OPEN ACCESS

Edited by: Barbara Zavan, University of Padua, Italy

Reviewed by:

Kaili Lin,

Shanghai Jiao Tong University, China

Yong-Can Huang,

Peking University Shenzhen Hospital,

China

${ }^{*}$ Correspondence:

Raorao Wang

raoraowang@hotmail.com

Yuanzhi Xu

amyxyz01@sina.com

Shengcai Q

dentistai@163.com

Specialty section:

This article was submitted to

Tissue Engineering and Regenerative

Medicine,

a section of the journa

Frontiers in Bioengineering and

Biotechnology

Received: 08 October 2020

Accepted: 08 January 2021

Published: 23 February 2021

Citation:

Zhao B, Peng Q, Poon EHL, Chen $F$, Zhou R, Shang $G$, Wang $D$,

Xu Y, Wang R and Qi S (2021)

Leonurine Promotes the Osteoblast

Differentiation of Rat BMSCs by

Activation of Autophagy via

the PI3K/Akt/mTOR Pathway.

Front. Bioeng. Biotechnol. 9:615191.

doi: 10.3389/fbioe.2021.615191

\section{Leonurine Promotes the Osteoblast Differentiation of Rat BMSCs by Activation of Autophagy via the PI3K/Akt/mTOR Pathway}

\author{
Bingkun Zhao', Qian Peng ${ }^{1}$, Enoch Hin Lok Poon ${ }^{2,3}$, Fubo Chen ${ }^{1}$, Rong Zhou', \\ Guangwei Shang ${ }^{1}$, Dan Wang ${ }^{2,3}$, Yuanzhi Xu ${ }^{1 *}$, Raorao Wang ${ }^{1 *}$ and Shengcai Qi ${ }^{1 *}$

\begin{abstract}
1 Department of Stomatology, Shanghai Tenth People's Hospital, School of Medicine, Tongji University, Shanghai, China,
${ }^{3}$ School of Biomedical Sciences, The Chinese University of Hong Kong, Hong Kong, China
\end{abstract} \\ 2 Institute for Tissue Engineering and Regenerative Medicine, The Chinese University of Hong Kong, Hong Kong, China,
}

Background: Leonurine, a major bioactive component from Herba leonuri, has been shown to exhibit anti-inflammatory and antioxidant effects. The aim of this study was to investigate the effect of leonurine on bone marrow-derived mesenchymal stem cells (BMSCs) as a therapeutic approach for treating osteoporosis.

Materials and Methods: Rat bone marrow-derived mesenchymal stem cells (rBMSCs) were isolated from 4-weeks-old Sprague-Dawley rats. The cytocompatibility of leonurine on rBMSCs was tested via CCK-8 assays and flow cytometric analyses. The effects of leonurine on rBMSC osteogenic differentiation were analyzed via ALP staining, Alizarin red staining, quantitative real-time polymerase chain reaction (qRT-PCR), and Western blot. Additionally, autophagy-related markers were examined via qRT-PCR and Western blot analyses of rBMSCs during osteogenic differentiation with leonurine and with or without 3-methyladenine (3-MA) as an autophagic inhibitor. Finally, the PI3K/Akt/mTOR signaling pathway was evaluated during rBMSC osteogenesis.

Results: Leonurine at 2-100 $\mu \mathrm{M}$ promoted the proliferation of rBMSCs. ALP and Alizarin red staining results showed that $10 \mu \mathrm{M}$ leonurine promoted rBMSC osteoblastic differentiation, which was consistent with the qRT-PCR and Western blot results. Compared with those of the control group, the mRNA and protein levels of Atg5, Atg7, and LC3 were upregulated in the rBMSCs upon leonurine treatment. Furthermore, leonurine rescued rBMSC autophagy after inhibition by 3-MA. Additionally, the PI3K/AKT/mTOR pathway was activated in rBMSCs upon leonurine treatment.

Conclusion: Leonurine promotes the osteoblast differentiation of rBMSCs by activating autophagy, which depends on the PI3K/Akt/mTOR pathway. Our results suggest that leonurine may be a potential treatment for osteoporosis.

Keywords: BMSCs, leonurine, autophagy, osteoporosis, PI3K/Akt/mTOR pathway 


\section{INTRODUCTION}

Osteoporosis is the most common bone disease and is found in one-tenth of the population aged above 50 years and onequarter of the population aged above 80 years (Wright et al., 2014). Concurrently, the rate of occurrence of osteoporosisrelated bone fractures is increasing; it is estimated that one in two women and one in five men aged above 50 years are at risk of bone fracture as a direct result of osteoporosis (Das and Crockett, 2013). This condition is a major clinical and public health concern, and high morbidity, disability, and mortality rates are associated with the disease. The risks of osteoporosis include age, inadequate calcium, vitamin D deficiency, female sex, lack of activity, smoking, alcohol consumption, and some chronic inflammatory diseases (Jing et al., 2016).

Osteoporosis is characterized by the fundamental imbalance between bone formation and resorption, which is caused by the overactivation of osteoclasts, an increase in cell apoptosis, and a decrease in the osteogenesis of BMSCs. This process results in a defining feature of osteoporosis: the loss of bone mass, which increases the risk of fracture. Inadequate bone formation by osteoblasts originating from bone marrow mesenchymal stem cells (BMSCs) to compensate for bone resorption by osteoclasts is considered the major cause of osteoporosis. Several studies have revealed that osteogenic differentiation is impaired in patients with osteoporosis and that recovering osteogenic capacities could treat osteoporosis (Jing et al., 2016).

Autophagy is a cellular process wherein the primary function is to clear damaged cellular components such as long-lived proteins and organelles, thus participating in conservation across multiple cell types. In pathological situations, autophagy plays an important role in maintaining bone homeostasis (PierrefiteCarle et al., 2015). Current evidence indicates the dysregulation of autophagy as a major contributor to the development of metabolic disorders, including insulin resistance, diabetes mellitus, obesity, atherosclerosis, and osteoporosis. Autophagy plays an integral role in maintaining bone homeostasis, with substantial evidence demonstrating its contribution to the survival of osteoblasts, regulation of osteoblast differentiation, maintenance of bone mass, and improvement of bone strength (Yao et al., 2016; Geng et al., 2019). In contrast, decreased autophagy leads to an increase in BMSC apoptosis and inhibition of osteoblast differentiation (Nollet et al., 2014). Recent literature demonstrates the relationship between autophagy and osteoblast differentiation: BMSCs contain many autophagosomes in their initial differentiation stage, illustrating a fundamental linkage between autophagy and metabolism involved in osteoblastic differentiation (Nuschke et al., 2014). This evidence provides a logical foundation for pursuing the treatment of osteoporosis via autophagic regulation in BMSCs.

Current medical treatments for osteoporosis commonly encompass diphosphonate, calcitonin, estrogen receptor modulators, and parathyroid hormone (PTH); accordingly, such treatments can cause undesired side effects, including gastrointestinal responses, renal toxicity (Xu et al., 2013), hypocalcemia (Smith et al., 2009), and an increased risk of cancer (Choi et al., 2020). For example, bisphosphonates trigger osteoclast apoptosis with therapeutic efficiency reaching bottlenecks at 3-5 years post-treatment, along with an increased risk of atypical femoral fractures (Han et al., 2017); Denosumab was reported to be correlated with atypical femoral fracture and osteonecrosis of the jaw, reported drug withdrawal symptoms, an increase risk of bone fractures due to a rapid increase in bone remodeling, and potential cardiovascular events (Adhikary et al., 2018); PTH was reported to induce abnormal psychiatric symptoms and a restricted anabolic window within 2 years of treatment (Lee et al., 2010). Alternatively, traditional Chinese medicine has been used to prevent and treat osteoporosis with a recorded history of over a thousand years; the use of herbal medicine and its extracts, including Epimedium, Salvia, Rehmanniae radix, and Ophiopognin, has been shown to elicit fewer side effects and demonstrated higher sustainability over long-term periods than synthetic drugs (Guo et al., 2014; Xu et al., 2016; Liu et al., 2017; Xi et al., 2018). Despite a deficit in the current understanding of drug-receptor interactions and signaling pathways involved in herbal medicine, traditional Chinese medicine still holds potential and empirical evidence identifies it as a safe and effective alternative in treating osteoporosis.

Leonurine is a natural chemical compound extracted from the traditional Chinese herbal medicine Herba leonuri. In particular, its high anti-inflammatory and antioxidant effects have been extensively illustrated and can reduce the damage caused by excessive ROS in many diseases, such as cardiovascular diseases, ischemic stroke, and atherosclerosis (Liu et al., 2010; Loh et al., 2010; Zhang et al., 2012). In motor system-related diseases, leonurine could contribute to the reconstruction of cartilage in disease situations and alleviate osteoporotic progression in a rat model by inhibiting osteoclast differentiation ( $\mathrm{Hu}$ et al., 2019; Chen et al., 2020). A recent study demonstrated that leonurine can regulate autophagy to ameliorate cognitive dysfunction (Liu et al., 2016). Therefore, we hypothesized that leonurine may be a selective medicine for targeting osteoporosis by regulating autophagic activity in BMSCs.

The aim of this study was to confirm the roles of leonurine in BMSCs and the underlying molecular mechanisms. The proliferation of BMSCs cocultured with leonurine was detected by CCK- 8 assays and flow cytometry in vitro. The osteogenic differentiation of leonurine on BMSCs was evaluated by ALP staining, PCR, and Western blot (WB) analysis. The $\mathrm{PI} 3 \mathrm{~K} / \mathrm{Akt} / \mathrm{mTOR}$ pathway was analyzed using WBs.

\section{MATERIALS AND METHODS}

\section{Cell Culture and Differentiation Assays}

Sprague-Dawley (SD) rat primary BMSCs were isolated from the bone marrow of 4-weeks-old rats. In brief, bone marrow cells were first flushed with cell culture medium and cultivated in Petri dishes. After $24 \mathrm{~h}$ of incubation, adherent cells (BMSCs) were extracted from each dish.

Extracted BMSCs were maintained in $\alpha$-MEM ( $\alpha$-MEM, HyClone, United States) with 10\% fetal bovine serum (FBS, Gibco, United States) and 1\% penicillin/streptomycin (PS, Gibco, 
United States). The cells were cultured at $37^{\circ} \mathrm{C} / 5 \% \mathrm{CO}_{2}$, and the medium was replaced every 3 days. With cells passaged upon reaching $80 \%$ confluency, BMSCs of passages 3-6 were used in the ensuing experiments.

For osteogenic differentiation, BMSCs were cultured with osteogenic induction medium containing $10 \%$ FBS, $1 \%$ penicillin/streptomycin, $50 \mu \mathrm{g} / \mathrm{ml}$ ascorbic acid (Sigma, United States), $10 \mathrm{mM}$ sodium $\beta$-glycerophosphate (Sigma, United States), and 10 nM dexamethasone (Sigma, United States).

\section{The Biocompatibility of Leonurine on BMSCs CCK8 (Cell Counting Kit-8) Assays}

Cell proliferation was measured by Cell Counting Kit-8 assays (CCK-8, Dojindo, Shanghai, China) according to the manufacturer's protocol with three replicates. The experimental group was treated with different concentrations of leonurine ( 0 $100 \mu \mathrm{M})$. Briefly, the cells were cultivated in 96-well plates at a density of $3 \times 10^{3}$ cells per well. The seeding density was measured after an initial overnight incubation with a CCK8 assay to test whether they were equal. Another repeat was performed after 3 days of culture, in which the medium was replaced with $10 \mu \mathrm{l}$ of CCK 8 solution dissolved in $200 \mu \mathrm{l}$ of cell culture medium. The plate was incubated for $2 \mathrm{~h}$ before absorbance at $450 \mathrm{~nm}$ was measured.

Flow Cytometric Analysis of Cell Cycle and Apoptosis BMSCs were seeded in six-well plates at a density of $2 \times 10^{4}$ cells per well and treated with leonurine for 3 days. Subsequently, the cells were collected and fixed with $75 \%$ ethanol overnight at $4{ }^{\circ} \mathrm{C}$ and washed with cold PBS twice before staining with 200 $\mu \mathrm{l}$ of PI/RNase staining buffer for $30 \mathrm{~min}$ at room temperature. Cell cycle distribution was detected by a BD FACSCanto II flow cytometer (BD BioScience, United States).

Apoptosis assays were further performed to ascertain the effect of leonurine on cell viability with FITC-Annexin V apoptosis detection kits (BD Bioscience, United States) according to the manufacturer's protocols. Briefly, the cells were collected, washed with cold PBS, and resuspended in $1 \times$ binding buffer. Then, they were stained with $5 \mu \mathrm{l}$ of Annexin V-FICT and $5 \mu \mathrm{M}$ propidium iodide (PI) in the dark for $15 \mathrm{~min}$ at room temperature. Cell apoptosis was detected by a BD FACSCanto II flow cytometer (BD BioScience, United States).

\section{The Osteogenic Differentiation of BMSCs Induced by Leonurine}

\section{Alkaline Phosphatase (ALP) Staining and Alizarin Red Staining}

BMSCs at a density of $2 \times 10^{4}$ cells/well were seeded in 24 well plates. After $24 \mathrm{~h}$, the baseline medium was replaced with osteogenic induction medium containing various concentrations of leonurine $(0,2,5$, and $10 \mu \mathrm{M})$. The cells were cultured in osteogenic induction medium for either 6 days (ALP staining) or 14 days (Alizarin red staining).
ALP staining was conducted to ascertain the effect of leonurine on BMSC differentiation. In brief, BMSCs were harvested after 6 days of culture and fixed with 4\% paraformaldehyde for $10 \mathrm{~min}$. An ALP color development kit (Beyotime, Shanghai, China) was used in the study according to the manufacturer's protocols. Briefly, the cells were stained for $15 \mathrm{~min}$ and washed three times with PBS. The stained cells were subsequently observed under phase-contrast microscopy, with representative images captured.

Alizarin red staining was further performed to determine the degree of calcium deposition in BMSCs between the leonurine treatment groups at various concentrations. Briefly, BMSCs were harvested after 14 days of culture in osteogenic medium as outlined above. After fixation in $4 \%$ paraformaldehyde for 10 min, the cells were stained with Alizarin red staining kits (Beyotime, Shanghai) for $60 \mathrm{~min}$ and washed three times with $\mathrm{ddH}_{2} \mathrm{O}$.

The stained cells were subsequently observed under phasecontrast microscopy, with representative images captured.

\section{RNA Isolation and Quantitative Real-Time PCR (qRT-PCR) Analysis}

BMSCs were seeded in six-well plates at a density of $5 \times 10^{4}$ cells/well. After $24 \mathrm{~h}$, osteogenic induction medium containing $10 \mu \mathrm{M}$ leonurine was added to the experimental groups, with the control groups receiving medium without leonurine. Total RNA from the BMSCs was isolated following osteogenic induction for 6 days with the TRIzol (Invitrogen, United States) extraction method according to the manufacturer's protocol. The total RNA concentration was measured by a Nanodrop system (Thermo Fisher Scientific, United States). cDNA was reverse transcribed with a PrimeScript RT reagent Kit (TaKaRa, Japan). qPCR was conducted with Hieff $^{\mathrm{TM}}{ }_{\mathrm{qPCR}} \mathrm{SYBR}^{\circledR}{ }^{\circledR}$ Green Master Mix in an ABI 7500 Real-Time PCR System (Applied Biosystems, Foster City, CA, United States). Relative gene expression was calculated using the comparative $2^{-\Delta \Delta C}$ method. The primers used are listed in Table 1.

TABLE 1 | Nucleotide sequences of primers used for qRT-PCR.

\begin{tabular}{lll}
\hline Gene & & Primer sequence(5'-3') \\
\hline OCN & Forward primer & TGAGGACCCTCTCTCTGCTC \\
OCN & Reverse primer & GGGCTCCAAGTCCATTGTT \\
OPN & Forward primer & ATCTGAGTCCTTCACTG \\
OPN & Reverse primer & GGGATACTGTTCATCAGAAA \\
RUNX2 & Forward primer & GCACCCAGCCCATAATAGA \\
RUNX2 & Reverse primer & TTGGAGCAAGGAGAACCC \\
p62/SQSTM1 & Forward primer & ACCCATCCACAGAGGCTGAT \\
p62/SQSTM1 & Reverse primer & GCCTTCATCCGAGAAACCCA \\
ATG5 & Forward primer & ACGTGTGGTTGGACGGATT \\
ATG5 & Reverse primer & AAGGCCGTTCAGTTGTGGTC \\
ATG7 & Forward primer & TGGAGCATGCCTACGATGAC \\
ATG7 & Reverse primer & TITGGGGTCCATACATCCGC \\
GAPDH & Forward primer & CAGGGCTGCCTTCTCTTGT \\
GAPDH & Reverse primer & TCCCGTTGATGACCAGCTTC
\end{tabular}




\section{Protein Extraction and WB Analysis}

BMSCs at a density of $5 \times 10^{4}$ cells/well were seeded in six-well plates and divided into a control group or treatment group (10 $\mu \mathrm{M}$ leonurine). Total protein from the rBMSCs was isolated following osteogenic induction for 6 days with RIPA buffer containing protease inhibitor and phosphatase inhibitor. Equal amounts of protein were separated and transferred onto nitrocellulose membranes (Millipore Corporation, Billerica, United States). Primary antibodies (GAPDH, OPG, and Runx2) were incubated with membranes at $4^{\circ} \mathrm{C}$ overnight, with the

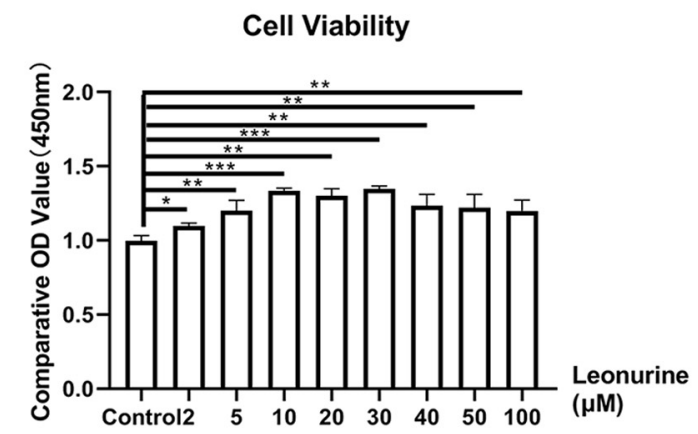

D

A

PI

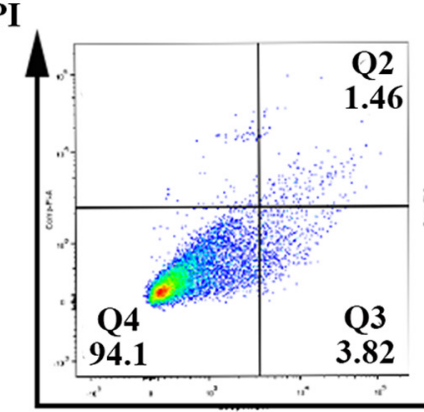

Control

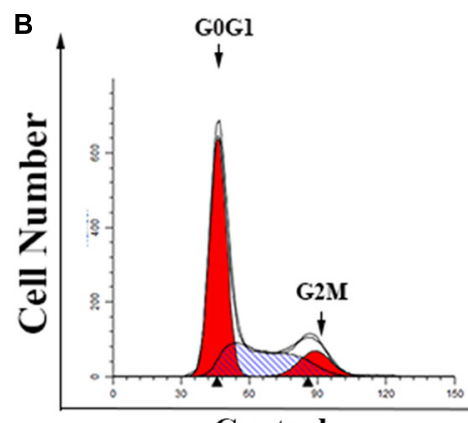

C

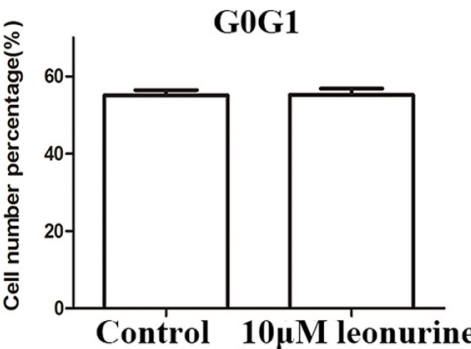

Control $10 \mu \mathrm{M}$ leonurine

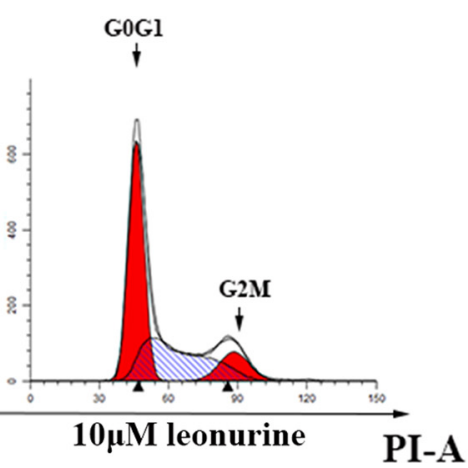

G2M

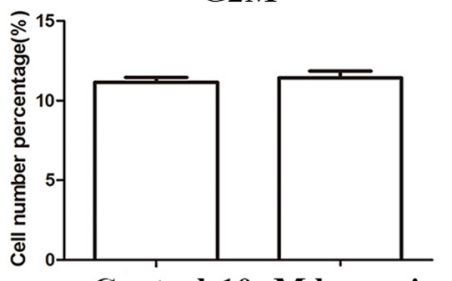

Control $10 \mu \mathrm{M}$ leonurine

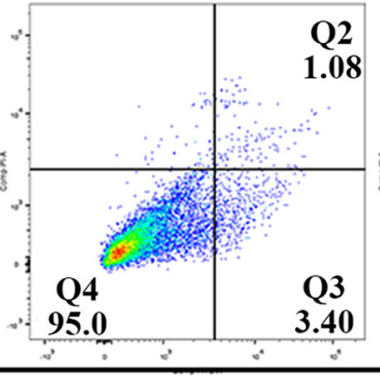

$2 \mu \mathrm{M}$ leonurine

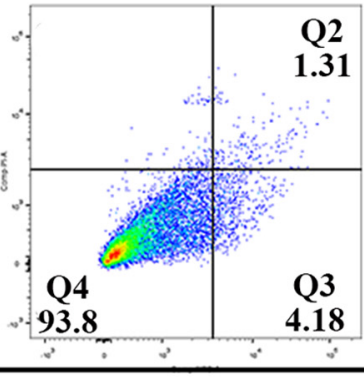

$5 \mu \mathrm{M}$ leonurine
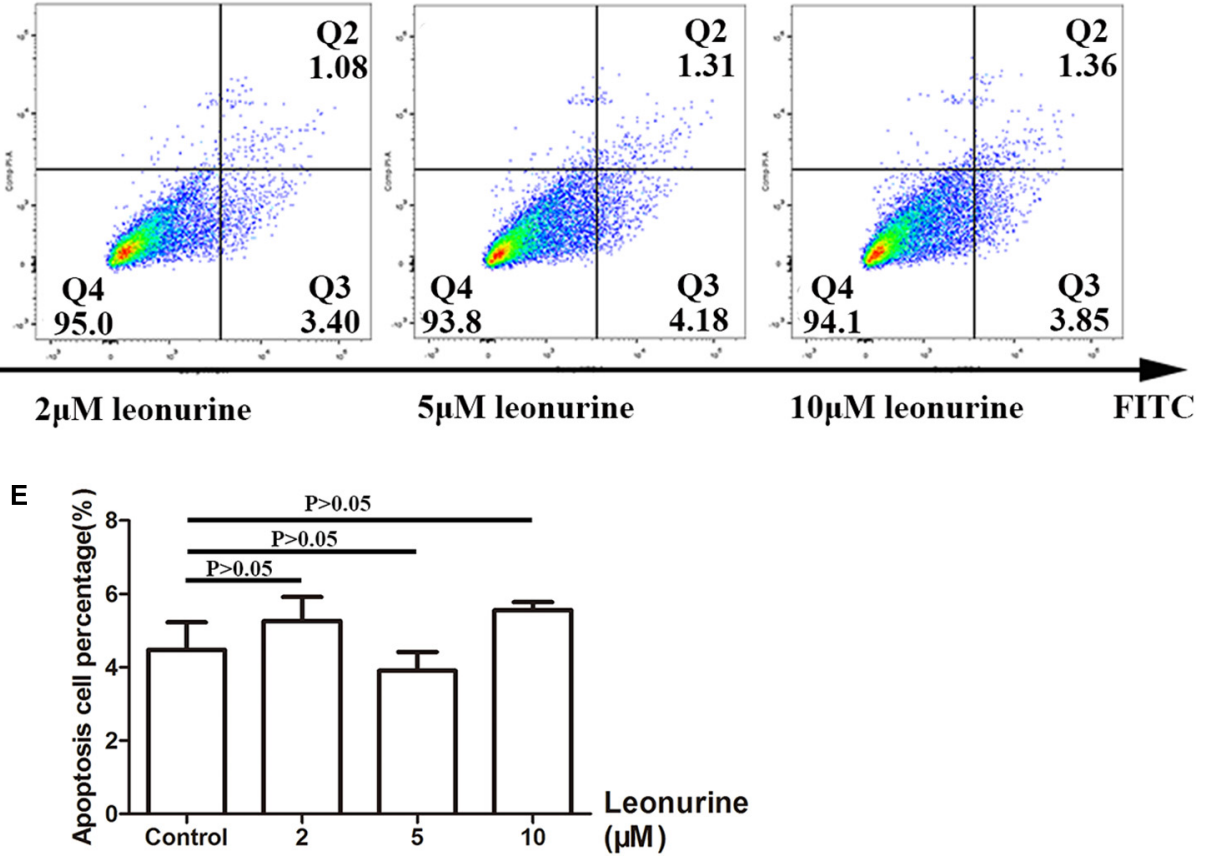

FIGURE 1 | Assessment of toxicity of leonurine and its effect on BMSC proliferation. (A) CCK-8 essay for BMSCs co-cultured with leonurine for 3 days illustrated increases in viability with leonurine-treated BMSCs. (B) Assessment of cell cycle phase distribution of leonurine -treated BMSCs. (C) Quantitative analysis of cell cycle phase distribution. No significant differences were measured between groups. (D) Distribution of apoptotic BMSCs observed under flow cytometry (FITC-Annexin V apoptotic detection assay). (E) Ratio of apoptotic BMSCs cultured in 0-10 $\mu$ M leonurine for 6 days. No significant differences were measured between groups $\left({ }^{\star} p<0.05,{ }^{\star \star} p<0.01,{ }^{\star \star \star} p<0.001\right)$. 
ensuing secondary antibody incubated at room temperature for $1 \mathrm{~h}$. The membranes were visualized using the Odyssey LI-CDR system. GAPDH $(1: 2,000)$ was purchased from Cell Signaling Technology (CST, Beverly, MA, United States). OPG (1:500) and Runx2 (1:500) were purchased from Abcam (Cambridge, MA, United States).

\section{The Effect of Leonurine on Autophagy-Related Genes}

BMSCs at a density of $5 \times 10^{4}$ cells/well were seeded in six-well plates. For autophagic inhibition, BMSCs were treated with $2 \mathrm{mM}$ 3-methyladenine (3-MA, Sigma, United States) with or without leonurine $(10 \mu \mathrm{M})$. First, 3-MA was dissolved in dimethyl sulfoxide (DMSO) prior to addition to the culture medium, with the final concentration of DMSO below $0.1 \%$ of the medium. Alizarin red staining was performed for 14 days and ALP staining was performed for 6 days as described above.

Additionally, WB and qPCR were performed with the no treatment (control) group, leonurine treatment group, 3-MA treatment group, and 3-MA with leonurine treatment group. The antibodies LC3AB I/II $(1: 1,000)$ and ATG7 (1:500) used in WB analyses were purchased from Cell Signaling Technology (CST, Beverly, MA, United States); P62 (1:1,000) was purchased from
Abcam (Cambridge, MA, United States). The primers used in the qPCR analysis are listed in Table $\mathbf{1 .}$

\section{Analysis of Pathways Related to Leonurine}

For PI3K activation, BMSCs were pretreated with $2 \mu \mathrm{M}$ of the PI3K activator 740Y-P (APExBIO, United States) for $2 \mathrm{~h}$ separately prior to coculturing with $10 \mu \mathrm{M}$ leonurine. Briefly, Y740Y-P was dissolved in DMSO, and the final concentration of DMSO contributed to less than $0.1 \%$ of the medium, inducing no notable cytotoxic effect. WB assays were performed as stated above. The experimental groups were the control group, pretreatment with the $740 \mathrm{Y}-\mathrm{P}$ group, leonurine treatment with the $740 \mathrm{Y}-\mathrm{P}$ pretreatment group, and the leonurine treatment group. Antibodies against AKT (1:1,000), p-AKT (1:1,000), and p-mTOR $(1: 1,000)$ were purchased from Cell Signaling Technology (CST, Beverly, MA, United States). Antibodies against PI3K $(1: 1,000)$ and $\mathrm{p}$-PI3K (1:500) were purchased from Abcam (Cambridge, MA, United States).

\section{Statistical Analysis}

Statistical analysis was performed by SPSS 20.0 (IBM, Somers, NY, United States). Each experiment was independently repeated
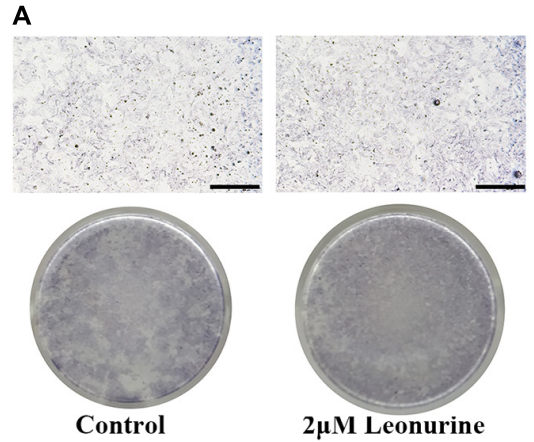

$2 \mu \mathrm{M}$ Leonurine

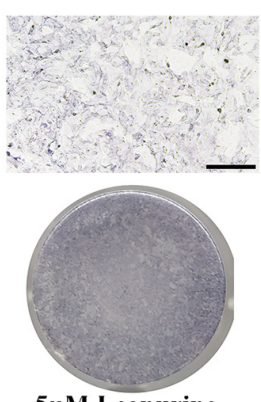

$5 \mu \mathrm{M}$ Leonurine

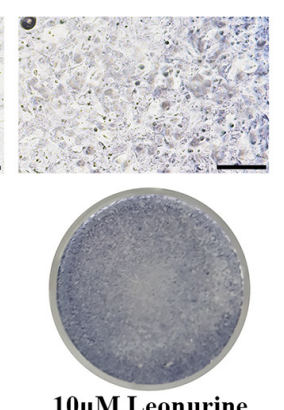

$10 \mu M$ Leonurine

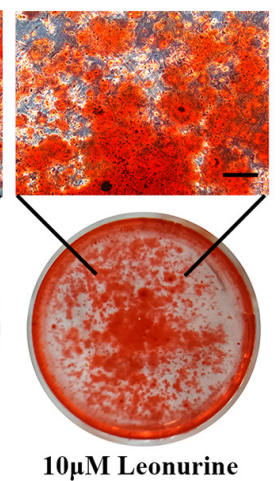

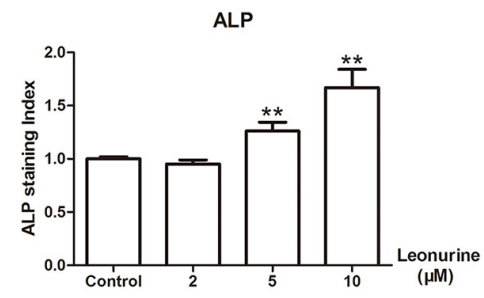

D

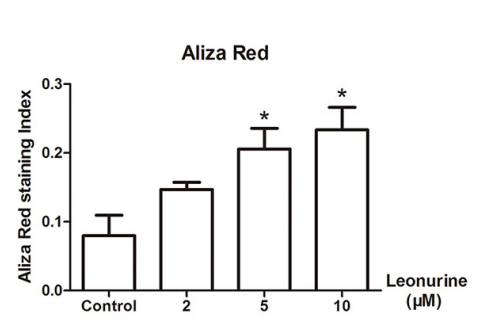

c

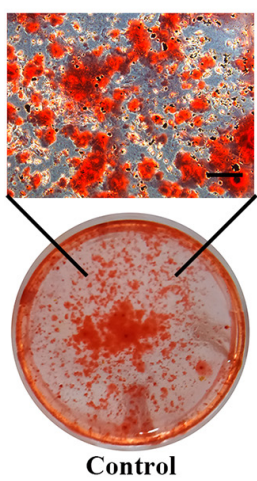

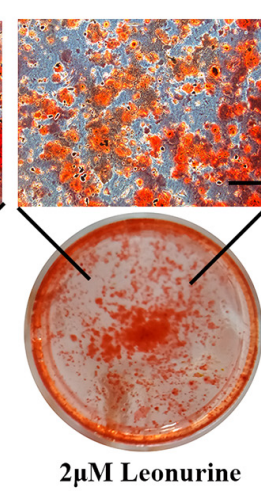

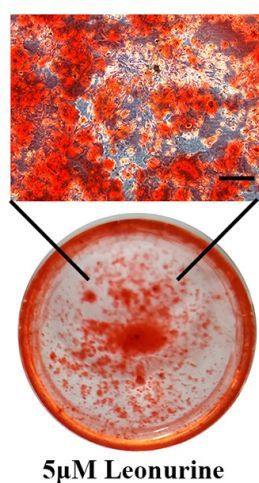

FIGURE 2 | Effects of leonurine on osteoblast differentiation and mineralization of BMSCs. (A) ALP staining of leonurine-treated groups (0-10 $\mu$ M) at day 6. (B) Quantitative analysis of ALP staining. Significant increases were recorded for 5 and $10 \mu \mathrm{M}$ leonurine-treated groups. (C) Aliza red staining of leonurine-treated groups $(0-10 \mu \mathrm{M})$ at day 14. (D) Quantitative analysis of ALP staining. Significant increases in mineralization were recorded for 5 and $10 \mu \mathrm{M}$ leonurine-treated group $\left({ }^{\star} p<0.05,{ }^{* *} p<0.01,{ }^{* \star *} p<0.001\right.$ vs. Control group). Scale bar $=500 \mu \mathrm{m}$. 
at least three times. Differences between two groups were analyzed with unpaired Student's $t$-test, and more than three groups were analyzed with one-way analysis of variance followed by the Bonferroni post-test. Data are presented as the mean \pm standard error (SEM). $P<0.05$ were considered to indicate significant differences.

\section{RESULTS}

\section{Leonurine Exhibits No Notable Toxicity and Contributes to BMSC Proliferation}

To assess the cytotoxicity of leonurine and its effect on the proliferation of BMSCs, we conducted a series of CCK- 8 cell viability assays, cell cycle distribution analyses, and apoptosis assays with flow cytometry. As shown in Figure 1A, our results demonstrated that no apparent cytotoxicity was observed in the leonurine-treated group after $72 \mathrm{~h}$ of coculture with BMSCs. In contrast, viability was significantly increased in the BMSCs treated with leonurine at a range of $2-100 \mu \mathrm{M}$, with a concentration of $10 \mu \mathrm{M}$ resulting in a peak increase in viability followed by a gradual decrease at higher concentrations (40-100 $\mu \mathrm{M})$. It reflects the fact that $10 \mu \mathrm{M}$ leonurine is the lowest and most proper functional concentration on BMSCs.

The flow cytometric results of BMSCs cocultured with leonurine demonstrated no notable change in the distribution of any particular cell cycle stage (Figures 1B,C). We observed an effect only on cell proliferation. However, whether the cell cycle period was changed was unclear. Apoptosis assays after coculture for 3 days further confirmed that leonurine did not have apparent toxicity and did not cause cell apoptosis (including early apoptosis and late apoptosis) (Figures 1D,E). These data proved the security of leonurine on BMSCs, and the lowest functional concentration is $10 \mu \mathrm{M}$.

\section{Leonurine Contributes to Osteoblast Differentiation}

To investigate the effect of leonurine on osteoblast differentiation, we performed ALP staining and Alizarin red staining after 6 and 14 days of culture, respectively, as an early marker for osteoblastic differentiation and a late marker for calcium deposition in osteoblasts.

As shown in Figure 2, leonurine contributed to osteoblastic differentiation in a dose-dependent manner, which was most apparent in the $10 \mu \mathrm{M}$ leonurine-treated group (Figure 2A), with a significant increase at day 6 (Figure 2B). Alizarin red staining yielded comparable results after 14 days of culture, where a significant increase in mineralization was recorded for the 10 $\mu \mathrm{M}$ leonurine-treated BMSCs (Figures 2C,D). Combined, these results demonstrated the significant effect of $10 \mu \mathrm{M}$ leonurine (compared to lower concentrations) in improving osteogenesis; we thereafter selected $10 \mu \mathrm{M}$ leonurine as the concentration for subsequent analyses.

\section{Leonurine Promotes Osteogenic Differentiation via the Activation of Autophagy}

To determine the contribution of leonurine to osteogenesis, we performed qRT-PCR and WB analysis to further quantify the results obtained via ALP and Alizarin red staining. As shown
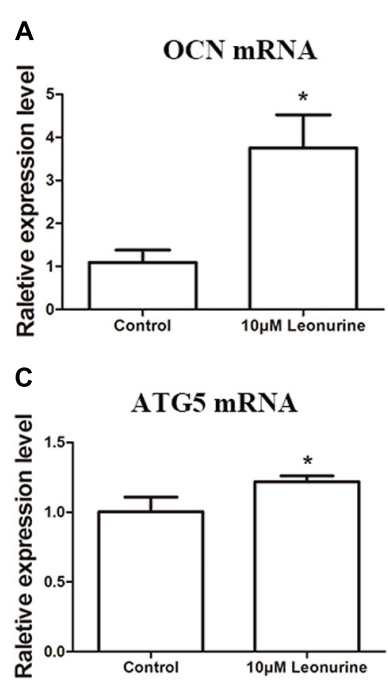
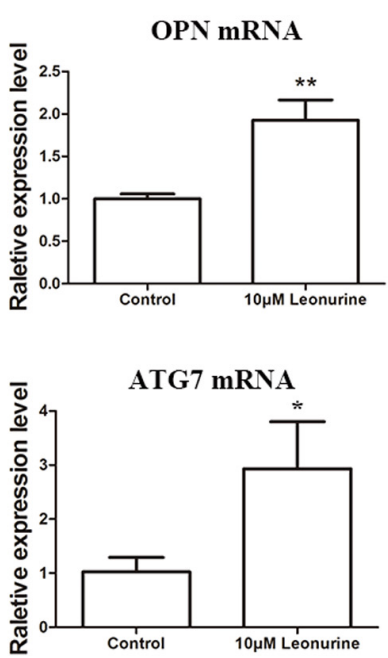

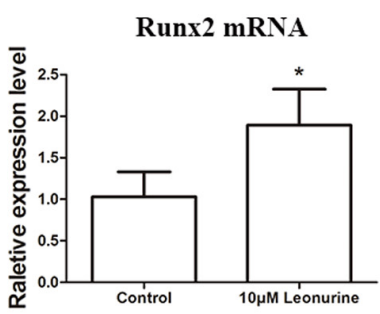

B
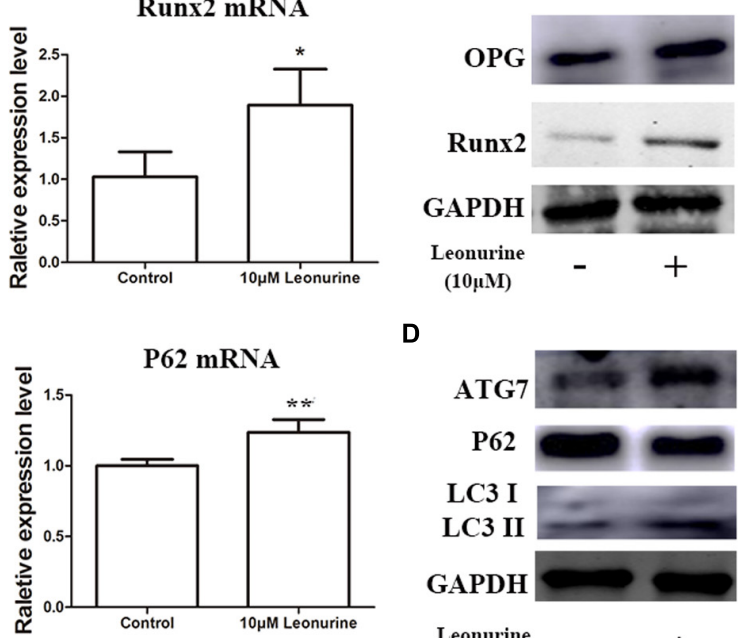

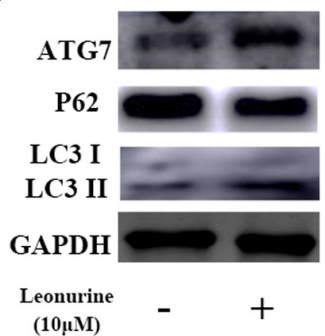

FIGURE 3 | Effects of leonurine on osteogenic and autophagic activity in BMSCs. (A) qRT-PCR analysis of osteogenesis related genes OCN, OPN, and Runx2 expression in BMSCs after 6 days of $10 \mu \mathrm{M}$ leonurine treatment. Expression of all 3 mRNAs were significantly increased compared to control. (B) Western blot analysis of osteogenesis related proteins OPG and Runx2 in BMSCs after 6 days of $10 \mu \mathrm{M}$ leonurine treatment. Both proteins were significantly increased post-treatment. (C) Expression of autophagy related genes ATG5, ATG7, and P62 in BMSCs after 6 days of $10 \mu \mathrm{M}$ leonurine treatment. Expression of all 3 mRNAs were significantly increased compared to control. (D) Expression of autophagy related proteins ATG7, P62, LC3 I, and LC3 II in BMSCs after 6 days of $10 \mu$ M leonurine treatment. Both proteins were significantly increased post-treatment $\left({ }^{\star} p<0.05,{ }^{\star \star} p<0.01,{ }^{\star \star \star} p<0.001\right.$ vs. Control group). 
in Figure 3, the addition of $10 \mu \mathrm{M}$ leonurine improved the expression of osteogenesis-related markers (OCN, OPN, and Runx2) at both the mRNA and protein levels (Figures 3A,B).

Owing to the significant correlation present between autophagic activity and osteogenesis, autophagy-related mRNA and proteins, including ATG7, P62, and LC3 I/II, were subsequently quantified to determine whether leonurine could significantly modulate autophagy in BMSCs. Both qRT-PCR and WB analyses demonstrated that autophagy was enhanced in the leonurine-treated BMSCs compared to the untreated control cells (Figures 3C,D). This finding indicates that leonurine activated autophagy while accelerating the osteogenic process, demonstrating a strong relationship between them.

To further investigate the correlation between leonurineinduced activation of autophagy and its contribution to osteogenesis, we applied the autophagic inhibitor 3-MA with or without coculture treatment with leonurine. qRT-PCR and WB analyses both demonstrated significant inhibition of osteogenic

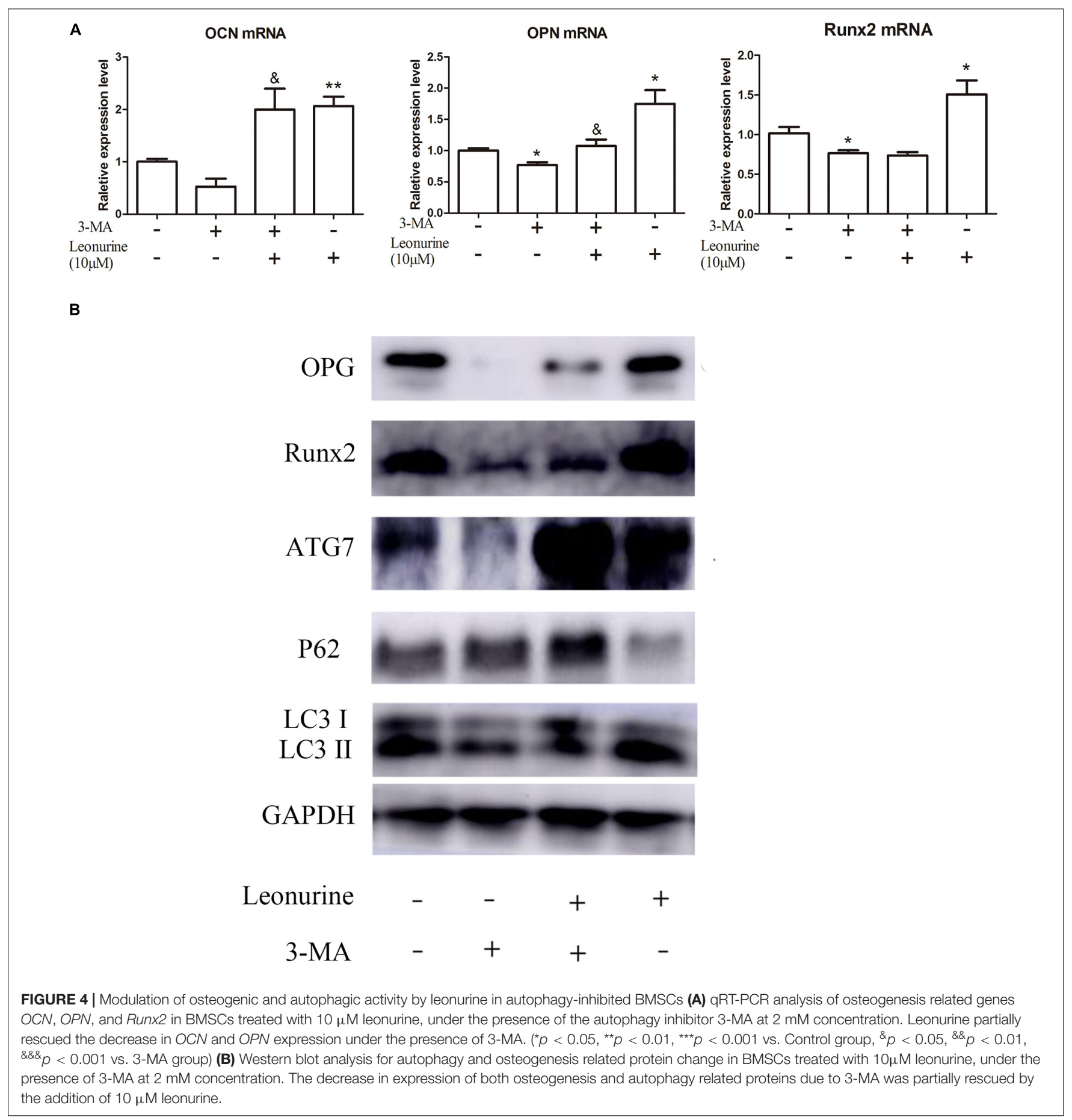


activity with 3-MA treatment (Figures 4A,B), indicating a decrease in osteogenesis via the inhibition of autophagy in BMSCs; concurrently, autophagy was partly rescued with leonurine supplemented with 3-MA (Figures 4A,B).

As additional evidence of the autophagic and osteogenic modulation of leonurine, significant inhibition of osteoblastic differentiation and mineralization by 3-MA was shown by ALP assays and Alizarin red staining after 6 and 14 days of culture, respectively, this phenomenon was reversed after the addition of $10 \mu \mathrm{M}$ leonurine (Figures $\mathbf{5 A}, \mathbf{C}$ ). The quantitative analysis is shown in Figures 5B,D.

As shown by this evidence, autophagic deficiency caused by 3-MA significantly decreased the osteogenic differentiation process, and these results support our hypothesis that leonurine can activate autophagy to contribute to osteogenesis.

\section{Leonurine Can Activate Autophagy by Inhibiting the PI3K/Akt/mTOR Pathway}

Considering the importance of the PI3K pathway involved in autophagy and leonurine previous report, we investigated the influence of leonurine on the PI3K pathway to ascertain its possible effect in modulating autophagy. Our results indicated that the PI3K activator activates PI3K within $2 \mathrm{~h}$, which is accompanied by the inhibition of autophagy (Figure 6A). Leonurine inhibited PI3K/Akt/mTOR activity with downregulation of phosphorylated PI3K/AKT/mTOR and exerted a negative effect against the PI3K activator 740-Y-P (Figure 6B). This evidence demonstrated that leonurine potentially activates autophagy via inhibition of the PI3K/Akt/mTOR pathway.

\section{DISCUSSION}

In this study, we demonstrated that leonurine, a natural compound derived from Leonurus, contributes to autophagy to improve BMSC differentiation without apparent cytotoxicity. Next, we further investigated its mechanism of autophagic activation by inhibiting the PI3K/Akt/mTOR pathway. Our results provide a rationale for developing leonurine as a new medicine for treatment of osteoporosis.

Many antiosteoporotic medicines result in various unavoidable side effects. In contrast, substantial evidence indicates a lowered risk of adverse events in treating osteoporosis

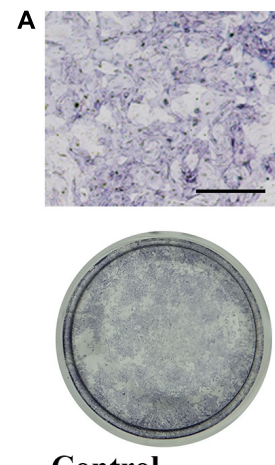

Control

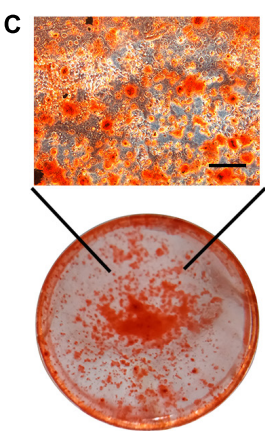

Control

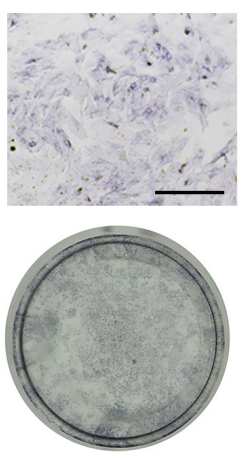

3-MA

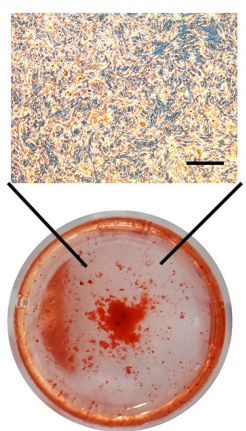

3-MA
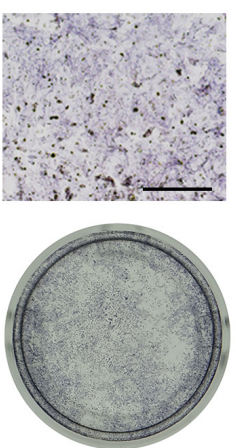

3-MA $10 \mu \mathrm{M}$ leonurine

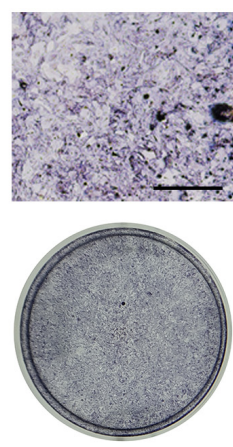

$10 \mu \mathrm{M}$ leonurine

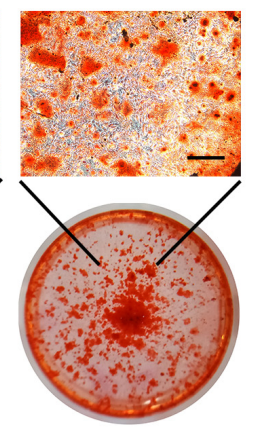

3-MA

$10 \mu \mathrm{M}$ leonurine

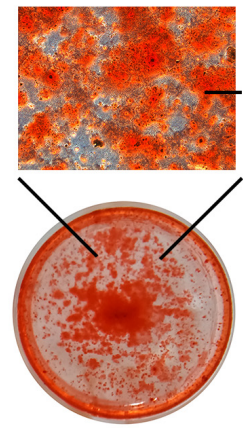

$10 \mu \mathrm{M}$ leonurine
B

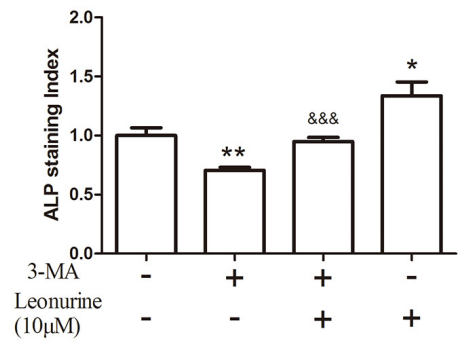

D

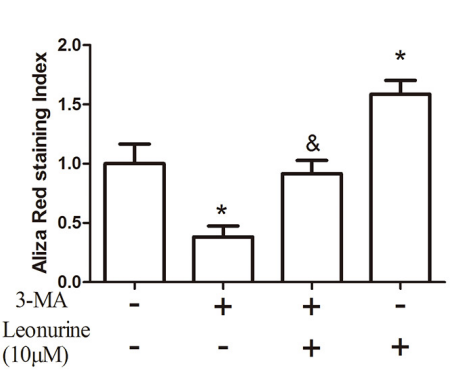

FIGURE 5 | Effects of leonurine on osteoblast differentiation and mineralization of autophagy-inhibited BMSCs. (A) ALP staining of 0-10 $\mu$ M leonurine treated BMSCs at day 6, with or without $2 \mathrm{mM}$ 3-MA for autophagy inhibition. $10 \mu \mathrm{M}$ leonurine partially reversed the inhibition of osteoclastic differentiation by 3-MA. (B) Quantitative analysis of ALP staining. (C) Alizarin red staining of 0-10 $\mu \mathrm{M}$ leonurine treated BMSCs at day 14, with or without 2 mM 3-MA for autophagy inhibition. $10 \mu \mathrm{M}$ leonurine partially reversed the inhibition of mineralization by 3-MA. (D) Quantitative analysis of Aliza red staining (Scale bar is $200 \mu \mathrm{M})\left({ }^{*} p<0.05\right.$, ${ }^{* \star} p<0.01,{ }^{\star \star \star} p<0.001$ vs. Control group, ${ }^{\&} p<0.05,{ }^{\&} \& p<0.01,{ }^{\&} \& \& p<0.001$ vs. 3-MA group). 

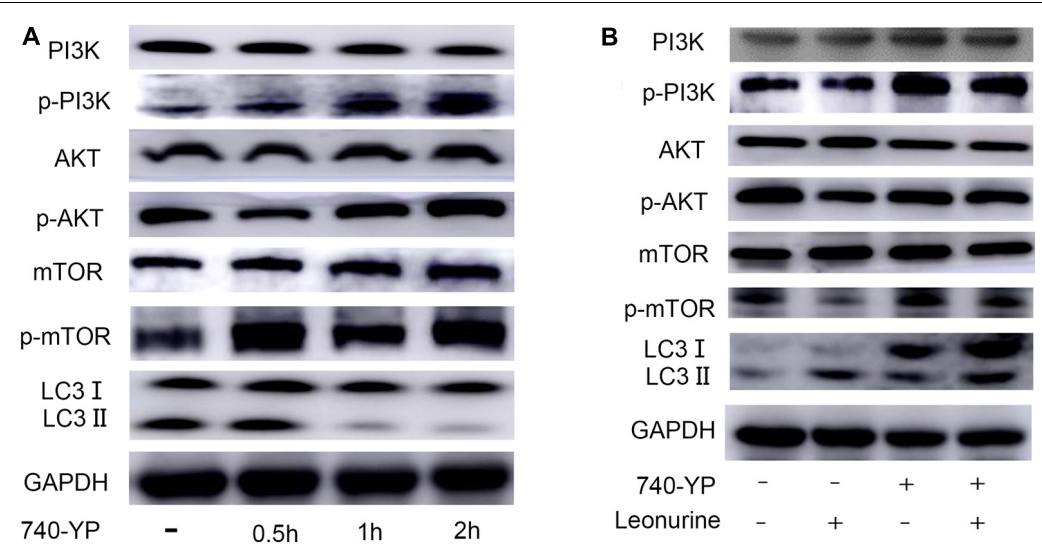

FIGURE 6 | Modulation of PI3K-AKT-mTOR pathway by leonurine. (A) Western blot analysis on the addition of the PI3K activator 740-YP in BMSCs. 740-YP at a concentration of $2 \mu \mathrm{M}$ increased expression of all downstream proteins in the PI3K-AKT-mTOR pathway. (B) Western blot analysis of the effect of leonurine on PI3K-AKT-mTOR pathway in BMSCs at $2 \mathrm{~h}$. Expression of downstream proteins in PI3K-AKT-mTOR pathway in BMSCs treated with or without $2 \mu \mathrm{M} 740-\mathrm{PP}$ was negatively regulated by the addition of $10 \mu \mathrm{M}$ leonurine.

with traditional Chinese medicine. Clinical studies have demonstrated that certain traditional Chinese medicines and their compounds can not only decrease bone resorption but also contribute to bone formation through estrogen-like effects, antioxidant activity, and modulation of bone metabolism (Zhang et al., 2016). For example, berberine promotes the differentiation of osteoblasts of BMSCs by stabilizing Runx2/Osterix through the increased activation of PKA signaling (Han et al., 2017). Kaempferol inhibits glucocorticoid-induced bone loss by promoting osteoblast survival through activation of the ERK pathway (Adhikary et al., 2018). Alisol-B suppresses RANKLinduced osteoclast formation to prevent bone loss through suppression of RANKL-mediated JNK pathway activation (Lee et al., 2010). Regarding the activity and effects of leonurine, it has been demonstrated that leonurine exhibits a protective function against cardiovascular disease, stroke, and nervous system disease by suppressing oxidative stress and chronic inflammation (Liu et al., 2013; Li et al., 2017). A recent study confirmed that leonurine has an antiosteoporotic effect on osteoclasts by inhibiting the PI3K-AKT and NF- $\kappa$ B signaling pathways (Yuan et al., 2015). To further elucidate this topic, we investigated the antiosteoporotic effect of leonurine on BMSC functional recovery. Our results illustrated that leonurine promoted the proliferation of BMSCs at appropriate concentrations. Concurrently, leonurine promoted bone mineralization, as shown by ALP staining and Alizarin red staining, along with upregulation of both osteogenic genes and proteins at a concentration of $10 \mu \mathrm{M}$. Combined, these results primarily indicated that leonurine can contribute to BMSC proliferation and differentiation, improving mineralization.

Osteoporosis associated with aging is characterized by consistent changes in bone metabolism with suppression of bone formation as well as increased bone resorption, both of which are associated with abnormal autophagic activity in osteoblasts (Bianco et al., 2011). Currently, there is substantial evidence to illustrate that autophagy can strongly contribute to osteoblast survival, regulate osteoblast differentiation, maintain bone mass, and improve bone strength : in detail, bone marrowderived mesenchymal stem cells have been regarded as the main contributors of osteoblasts to bone formation (Noort et al., 2002). With increasing age, bone marrow-derived mesenchymal stem cells tend to lose their self-renewal capacity and are directed toward adipogenic differentiation instead of osteogenesis; this phenomenon contributes significantly to bone loss and lipid accumulation in bone marrow (Moerman et al., 2004). Related studies have revealed that autophagy is a necessary factor for maintaining stemness and differentiation and that defective autophagy contributes to a decline in both cell count and cellular functions (Garcia-Prat et al., 2016).

The decline in autophagic activity is strongly correlated with osteoporosis, in which the inhibition of autophagy leads to increased cell apoptosis and activation of autophagy contributes to increased cell viability (Yang et al., 2014). Previous studies have demonstrated that knockout of the autophagy-related genes $B E C N-1, A T G 7$, and $L C 3$ results in defective bone mineralization; concurrently, enhancing autophagy helps BMSCs retain their multipotency (Sargolzaeiaval et al., 2018). Prior research has examined the contribution of leonurine to the regulation of autophagy. In this study, we demonstrated that leonurine successfully activated autophagy in BMSCs and increased the expression of autophagy-related proteins during the BMSC differentiation stage. Following leonurine administration, the impaired osteogenic differentiation in BMSCs induced by the autophagic inhibitor 3-MA was partially recovered. Combined, this evidence indicates that leonurine could regulate BMSC function by activating autophagy.

Substantial evidence has confirmed the relationship between dysregulated autophagy and osteoporosis in vitro and in vivo (Liu et al., 2018). Based on these studies, autophagic activation was shown to contribute to osteogenesis and serves as a promising target in treating osteoporosis. Many studies have investigated autophagy-related pathways. Among them, 
mTOR-related pathways, including PI3K/Akt/mTOR, are highly involved in the regulation of cell autophagy (Gao et al., 2016), and evidence identifies the mTOR signaling pathway as a modulatory factor in mediating human osteoblastic differentiation. Deletion of Raptor, an essential component of the mTORC1 gene, in osteocytes did not affect bone development and growth but led to increased trabecular bone mass (Liu et al., 2018). Relevant studies have indicated that suppressing the phosphorylation of mTOR leads to the activation of autophagy, concurrently eliciting antiapoptotic effects on BMSCs and osteoblasts (Yang et al., 2019). Furthermore, the latest research indicates that suppression of the PI3K/AKT/mTOR pathway is a protective factor in glucocorticoid-induced osteoporosis (Wang et al., 2020).

Recent research has shown that leonurine can inhibit RANKL-mediated osteoclastogenesis, reducing the loss of bone volume caused by estrogen deficiency, and evidence of its antiosteoporotic function via upregulated osteogenesis in BMSCs was presented. Our overall results support the hypothesis that leonurine promotes BMSC osteoblastic differentiation via its main action of autophagic activation through PI3K/Akt/mTOR pathway inhibition. Previous literature has demonstrated the successful modulation of the PI3K/Akt/mTOR pathway by leonurine in various diseases (Loh et al., 2010; Yuan et al., 2015). In another study, leonurine inhibited PI3K/Akt/mTOR pathway activation, and the results strongly indicated direct binding to the PI3K protein in chondrocyte cells. Therefore, there is a strong relationship between PI3K/Akt/mTOR and autophagy. We first clarified that leonurine induced inhibition of the PI3K pathway, which has a direct relationship with autophagy. Comparable results were found in our research, in which PI3K phosphorylation was inhibited after leonurine treatment. Concurrently, our results suggested that leonurine induces a negative effect on the PI3K/AKT/mTOR pathway, acting as a direct antagonist of the PI3K/AKT activator (740Y$\mathrm{P})$-dependent signaling pathway in this study. This finding contributes to our understanding of the mechanism by which leonurine harnesses autophagic activity to stimulate osteogenesis. However, a majority of other research on the leonurine-involved pathway has mainly focused on the NF- $\kappa \mathrm{B}$ pathway, which has a strong relationship with inflammation (Hoesel and Schmid, 2013). Osteoporosis-related research on leonurine reported that leonurine impedes osteoclasts differentiation by inhibition of PI3K/AKT and NF-кB pathway (Yuan et al., 2015). We combined the evidence of direct combination to PI3K protein to further test leonurine mechanism of $\mathrm{PI} 3 \mathrm{~K} / \mathrm{Akt} / \mathrm{mTOR}$ pathway regulation in osteoblasts. This compensates the research on anti-osteoporosis

\section{REFERENCES}

Adhikary, S., Choudhary, D., Ahmad, N., Karvande, A., Kumar, A., Banala, V. T., et al. (2018). Dietary flavonoid kaempferol inhibits glucocorticoid-induced bone loss by promoting osteoblast survival. Nutrition 53, 64-76. doi: 10.1016/j.nut.2017. 12.003

Bianco, P., Sacchetti, B., and Riminucci, M. (2011). Stem cells in skeletal physiology and endocrine diseases of bone. Endocr. Dev. 21, 91-101. doi: $10.1159 / 000328138$ mechanism from another aspect. However, our research did not examine NF-кB pathway function in autophagy, and this crosstalk with the PI3K pathway should further be taken into consideration for analysis of the mechanism of leonurine in a future study.

In conclusion, our research suggests the possible use of leonurine in activating BMSC autophagy to treat osteoporosis. Leonurine inhibits the PI3K/Akt/mTOR pathway to activate autophagy, subsequently contributing to osteoblast differentiation. These results strongly suggest that leonurine is a candidate medicine for potential studies in developing new therapies for osteoporosis.

\section{DATA AVAILABILITY STATEMENT}

The original contributions presented in the study are included in the article, further inquiries can be directed to the corresponding authors.

\section{AUTHOR CONTRIBUTIONS}

SQ designed the experiment and was responsible for the reviewers' suggestion. RW helped designed the experiments and checked uploaded data and rearranged the documents. YX and RW funded the experiments. BZ carried out the experiment and wrote the manuscript. QP, RZ, and GS were assistant of experiments. DW and EP helped the language edition. All authors contributed to the article and approved the submitted version.

\section{FUNDING}

This research was funded by the Science and Technology Commission of Shanghai Municipality, grant no. 03.02.18.003 and the Shanghai Municipal Health Commission, grant no. 20ZR1443100.

\section{ACKNOWLEDGMENTS}

We give thanks to Prof. Yizhun Zhu from the Department of Pharmacology, State Key Laboratory of Quality Research in Chinese Medicine and School of Pharmacy, Macau University of Science and Technology, for providing leonurine.

Chen, C., Zhu, Z., Hu, N., Liang, X., and Huang, W. (2020). Leonurine hydrochloride suppresses inflammatory responses and ameliorates cartilage degradation in osteoarthritis via NF-kappaB signaling pathway. Inflammation 43, 146-154. doi: 10.1007/s10753-019-01104-z

Choi, D., Choi, S., Chang, J., and Park, S. M. (2020). Exposure to oral bisphosphonates and risk of gastrointestinal cancer. Osteoporos. Int. 31, 775782. doi: 10.1007/s00198-020-05327-x

Das, S., and Crockett, J. C. (2013). Osteoporosis - a current view of pharmacological prevention and treatment. Drug Des. Devel. Ther. 7, 435-448. doi: 10.2147/ dddt.s31504 
Gao, J., Cheng, T. S., Qin, A., Pavlos, N. J., Wang, T., Song, K., et al. (2016). Glucocorticoid impairs cell-cell communication by autophagy-mediated degradation of connexin 43 in osteocytes. Oncotarget 7, 26966-26978. doi: 10.18632 /oncotarget.9034

Garcia-Prat, L., Martinez-Vicente, M., Perdiguero, E., Ortet, L., Rodriguez-Ubreva, J., Rebollo, E., et al. (2016). Autophagy maintains stemness by preventing senescence. Nature 529, 37-42. doi: 10.1038/nature16187

Geng, W., Shi, H., Zhang, X., Tan, W., Cao, Y., and Mei, R. (2019). Substance P enhances BMSC osteogenic differentiation via autophagic activation. Mol. Med. Rep. 20, 664-670.

Guo, Y., Li, Y., Xue, L., Severino, R. P., Gao, S., Niu, J., et al. (2014). Salvia miltiorrhiza: an ancient Chinese herbal medicine as a source for antiosteoporotic drugs. J. Ethnopharmacol. 155, 1401-1416. doi: 10.1016/j.jep.2014. 07.058

Han, Y., Jin, Y., Lee, S. H., Khadka, D. B., Cho, W. J., and Lee, K. Y. (2017). Berberine bioisostere Q8 compound stimulates osteoblast differentiation and function in vitro. Pharmacol. Res. 119, 463-475. doi: 10.1016/j.phrs.2017.03.002

Hoesel, B., and Schmid, J. A. (2013). The complexity of NF-kappaB signaling in inflammation and cancer. Mol. Cancer 12:86. doi: 10.1186/1476-4598-12-86

Hu, Z. C., Gong, L. F., Li, X. B., Fu, X., Xuan, J. W., Feng, Z. H., et al. (2019). Inhibition of PI3K/Akt/NF-kappaB signaling with leonurine for ameliorating the progression of osteoarthritis: in vitro and in vivo studies. J Cell Physiol. 234, 6940-6950. doi: $10.1002 /$ jcp. 27437

Jing, H., Liao, L., An, Y., Su, X., Liu, S., Shuai, Y., et al. (2016). Suppression of EZH2 prevents the shift of osteoporotic MSC fate to adipocyte and enhances bone formation during Osteoporosis. Mol. Ther. 24, 217-229. doi: 10.1038/mt. 2015.152

Lee, J. W., Kobayashi, Y., Nakamichi, Y., Udagawa, N., Takahashi, N., Im, N. K., et al. (2010). Alisol-B, a novel phyto-steroid, suppresses the RANKL-induced osteoclast formation and prevents bone loss in mice. Biochem. Pharmacol. 80, 352-361. doi: 10.1016/j.bcp.2010.04.014

Li, N., Xu, Q., Liu, Q., Pan, D., Jiang, Y., Liu, M., et al. (2017). Leonurine attenuates fibroblast-like synoviocyte-mediated synovial inflammation and joint destruction in rheumatoid arthritis. Rheumatology 56, 1417-1427. doi: 10.1093/rheumatology/kex142

Liu, C., Ma, R., Wang, L., Zhu, R., Liu, H., Guo, Y., et al. (2017). Rehmanniae Radix in osteoporosis: a review of traditional Chinese medicinal uses, phytochemistry, pharmacokinetics and pharmacology. J. Ethnopharmacol. 198, 351-362. doi: 10.1016/j.jep.2017.01.021

Liu, C., Yin, H., Gao, J., Xu, X., Zhang, T., and Yang, Z. (2016). Leonurine ameliorates cognitive dysfunction via antagonizing excitotoxic glutamate insults and inhibiting autophagy. Phytomedicine 23, 1638-1646. doi: 10.1016/j. phymed.2016.10.005

Liu, Q., Liu, C., Yang, Y., Yang, H., and Chen, J. (2018). Osteocyte-intrinsic mTORC1 signaling restrains trabecular bone accrual in mice. J. Cell Biochem. 119, 8743-8749. doi: $10.1002 /$ jcb. 27470

Liu, X. H., Pan, L. L., Chen, P. F., and Zhu, Y. Z. (2010). Leonurine improves ischemia-induced myocardial injury through antioxidative activity. Phytomedicine 17, 753-759. doi: 10.1016/j.phymed.2010.01.018

Liu, X. H., Pan, L. L., Deng, H. Y., Xiong, Q. H., Wu, D., Huang, G. Y., et al. (2013). Leonurine (SCM-198) attenuates myocardial fibrotic response via inhibition of NADPH oxidase 4. Free Radic. Biol. Med. 54, 93-104. doi: 10.1016/j. freeradbiomed.2012.10.555

Loh, K. P., Qi, J., Tan, B. K., Liu, X. H., Wei, B. G., and Zhu, Y. Z. (2010). Leonurine protects middle cerebral artery occluded rats through antioxidant effect and regulation of mitochondrial function. Stroke 41, 2661-2668. doi: 10.1161/strokeaha.110.589895

Moerman, E. J., Teng, K., Lipschitz, D. A., and Lecka-Czernik, B. (2004). Aging activates adipogenic and suppresses osteogenic programs in mesenchymal marrow stroma/stem cells: the role of PPAR-gamma2 transcription factor and TGF-beta/BMP signaling pathways. Aging Cell 3, 379-389. doi: 10.1111/j.14749728.2004.00127.x

Nollet, M., Santucci-Darmanin, S., Breuil, V., Al-Sahlanee, R., Cros, C., Topi, M., et al. (2014). Autophagy in osteoblasts is involved in mineralization and bone homeostasis. Autophagy 10, 1965-1977. doi: 10.4161/auto.36182

Noort, W. A., Kruisselbrink, A. B., In’T, A. P., Kruger, M., van Bezooijen, R. L., de Paus, R. A., et al. (2002). Mesenchymal stem cells promote engraftment of human umbilical cord blood-derived CD34(+) cells in NOD/SCID mice. Exp. Hematol. 30, 870-878. doi: 10.1016/s0301-472x(02)00820-2
Nuschke, A., Rodrigues, M., Stolz, D. B., Chu, C. T., Griffith, L., and Wells, A. (2014). Human mesenchymal stem cells/multipotent stromal cells consume accumulated autophagosomes early in differentiation. Stem Cell Res. Ther. 5:140. doi: $10.1186 /$ scrt530

Pierrefite-Carle, V., Santucci-Darmanin, S., Breuil, V., Camuzard, O., and Carle, G. F. (2015). Autophagy in bone: self-eating to stay in balance. Age. Res. Rev. 24, 206-217. doi: 10.1016/j.arr.2015.08.004

Sargolzaeiaval, F., Zhang, J., Schleit, J., Lessel, D., Kubisch, C., Precioso, D. R., et al. (2018). CTC1 mutations in a Brazilian family with progeroid features and recurrent bone fractures. Mol. Genet. Genom. Med. 6, 1148-1156. doi: 10.1002/mgg3.495

Smith, M. R., Egerdie, B., Hernandez, T. N., Feldman, R., Tammela, T. L., Saad, F., et al. (2009). Denosumab in men receiving androgen-deprivation therapy for prostate cancer. N. Engl. J. Med. 361, 745-755.

Wang, X. Y., Gong, L. J., Huang, J. M., Jiang, C., and Yan, Z. Q. (2020). Pinocembrin alleviates glucocorticoid-induced apoptosis by activating autophagy via suppressing the PI3K/Akt/mTOR pathway in osteocytes. Eur. J. Pharmacol. 880:173212. doi: 10.1016/j.ejphar.2020.173212

Wright, N. C., Looker, A. C., Saag, K. G., Curtis, J. R., Delzell, E. S., Randall, S., et al. (2014). The recent prevalence of osteoporosis and low bone mass in the United States based on bone mineral density at the femoral neck or lumbar spine. J. Bone Miner. Res. 29, 2520-2526. doi: 10.1002/jbmr.2269

Xi, H. R., Ma, H. P., Yang, F. F., Gao, Y. H., Zhou, J., Wang, Y. Y., et al. (2018). Total flavonoid extract of epimedium herb increases the peak bone mass of young rats involving enhanced activation of the AC10/cAMP/PKA/CREB pathway. J. Ethnopharmacol. 223, 76-87. doi: 10.1016/j.jep.2018.05.023

Xu, F., Ding, Y., Guo, Y., Liu, B., Kou, Z., Xiao, W., et al. (2016). Anti-osteoporosis effect of Epimedium via an estrogen-like mechanism based on a system-level approach. J. Ethnopharmacol. 177, 148-160. doi: 10.1016/j.jep.2015.11.007

Xu, X. L., Gou, W. L., Wang, A. Y., Wang, Y., Guo, Q. Y., Lu, Q., et al. (2013). Basic research and clinical applications of bisphosphonates in bone disease: what have we learned over the last 40 years? J. Transl. Med. 11:303. doi: 10.1186/1479-5876-11-303

Yang, X., Jiang, T., Wang, Y., and Guo, L. (2019). The role and mechanism of SIRT1 in resveratrol-regulated osteoblast autophagy in osteoporosis rats. Sci. Rep. 9:18424.

Yang, Y. H., Li, B., Zheng, X. F., Chen, J. W., Chen, K., Jiang, S. D., et al. (2014). Oxidative damage to osteoblasts can be alleviated by early autophagy through the endoplasmic reticulum stress pathway-implications for the treatment of osteoporosis. Free Radic. Biol. Med. 77, 10-20. doi: 10.1016/j.freeradbiomed. 2014.08.028

Yao, W., Dai, W., Jiang, L., Lay, E. Y., Zhong, Z., Ritchie, R. O., et al. (2016). Sclerostin-antibody treatment of glucocorticoid-induced osteoporosis maintained bone mass and strength. Osteoporos. Int. 27, 283-294. doi: 10.1007/ s00198-015-3308-6

Yuan, F. L., Xu, R. S., Jiang, D. L., He, X. L., Su, Q., Jin, C., et al. (2015). Leonurine hydrochloride inhibits osteoclastogenesis and prevents osteoporosis associated with estrogen deficiency by inhibiting the NF-kappaB and PI3K/Akt signaling pathways. Bone 75, 128-137. doi: 10.1016/j.bone.2015.02.017

Zhang, N. D., Han, T., Huang, B. K., Rahman, K., Jiang, Y. P., Xu, H. T., et al. (2016). Traditional Chinese medicine formulas for the treatment of osteoporosis: Implication for antiosteoporotic drug discovery. J. Ethnopharmacol. 189, 61-80. doi: 10.1016/j.jep.2016.05.025

Zhang, Y., Guo, W., Wen, Y., Xiong, Q., Liu, H., Wu, J., et al. (2012). SCM198 attenuates early atherosclerotic lesions in hypercholesterolemic rabbits via modulation of the inflammatory and oxidative stress pathways. Atherosclerosis 224, 43-50. doi: 10.1016/j.atherosclerosis.2012.06.066

Conflict of Interest: The authors declare that the research was conducted in the absence of any commercial or financial relationships that could be construed as a potential conflict of interest.

Copyright (๑) 2021 Zhao, Peng, Poon, Chen, Zhou, Shang, Wang, Xu, Wang and Qi. This is an open-access article distributed under the terms of the Creative Commons Attribution License (CC BY). The use, distribution or reproduction in other forums is permitted, provided the original author(s) and the copyright owner(s) are credited and that the original publication in this journal is cited, in accordance with accepted academic practice. No use, distribution or reproduction is permitted which does not comply with these terms. 